\title{
Ex vivo veritas
}

\section{Jean-Claude Kaplan}

Professeur émérite à la Faculté de Médecine Paris Descartes,

Institut Cochin, 24, rue du Faubourg Saint-Jacques,

75014 Paris, France.

kaplan@cochin.inserm.fr

Ayant eu le privilège d'assister au Symposium International sur les cellules \&S humaines (FISH-ESC) organisé par Marc Peschanski (Genocentre d'Évry, 31 janvier-2 février 2008) $\rightarrow$, je voudrais le replacer dans

\section{$(\rightarrow)$ Voir le compte rendu de L. Coulombel, p. 419 de ce numéro}

le contexte de la révolution de la biologie moléculaire et de son impact médical. La plupart des gènes de maladies humaines et leurs mutations pathogènes ont été identifiés; le génome humain et celui de très nombreux organismes du haut en bas de l'échelle du monde vivant ont été séquencés ; il est désormais possible d'analyser la fonction des séquences génomiques (on n'ose plus parler de «gènes »), et de reconstituer les réseaux d'interaction de leurs produits transcriptionnels et traductionnels (on n'ose plus dire «ARN » et «protéines»). Pourtant, si l'on se place au niveau nécessairement utilitariste de la médecine, il faut convenir que les retombées thérapeutiques n'ont pas été à la mesure des progrès cognitifs. En particulier en matière de thérapie génique, prématurément considérée comme une panacée, et inconsidérément présentée aux patients comme une voie royale. Pouvoir guérir aujourd'hui le lymphome de Hodgkin, dont on ne sait rien du mécanisme, et ne pas pouvoir guérir la drépanocytose dont tous les mécanismes sont élucidés depuis longtemps, n'est pas un moindre paradoxe Ce n'est pas une raison pour se laisser aller au blues du génomiste ${ }^{1}$. En effet, il existe une nouvelle frontière en matière de biothérapie, c'est la biologie «ex vivo», intermédiaire obligé entre l'in vitro et l'in vivo. Autrement dit, il s'agit de remettre la cellule vivante au premier plan des préoccupations, en profitant de la masse des connaissances accumulées en génomique et en protéomique, et à la lumière des percées décisives qu'ont été la création des premières lignées de cellules \&S murines (1981), et la découverte de la reprogrammation nucléaire (Dolly, 1997). Pouvoir repeupler un tissu, un organe, un organisme entier à partir de cellules souches hétérologues ou mieux autologues, voilà l'objectif de ce que l'on

${ }^{1}$ «L'ADN est triste et j'ai lu tous les génomes ». appelle désormais la «médecine régénérative ». Celleci dépend de la maîtrise des procédés de culture et de la connaissance des facteurs de conditionnement qui modulent la dynamique du développement et de la différenciation depuis l'état de zygote monocellulaire jusqu'à l'organisme adulte. Trois étapes ont été décisives dans ce domaine: (1) l'obtention en 1998 des premières lignées \&S humaines; (2) l'assouplissement des contraintes réglementaires d'ordre éthique concernant les recherches sur les cellules embryonnaires humaines (en France à partir de 2004) ; (3) la découverte en 2007 des facteurs biochimiques permettant de déprogrammer les cellules somatiques humaines différenciées pour les faire retomber dans l'enfance de cellules souches plus ou moins pluripotentes (les fameuses iPS).

Ce premier symposium international sur les cellules ES a réuni un panel des meilleurs spécialistes mondiaux. Ceux-ci ont fait l'inventaire des obstacles, et laissé entrevoir des pistes cognitives et technologiques pour les surmonter. Ils ont surtout montré les bénéfices que l'on pouvait raisonnablement escompter de la biologie ex vivo: modélisation des pathologies; découvertes de cibles et de cribles thérapeutiques; maîtrise des cultures massives de cellules, uniformément bloquées à un stade déterminé, puis mise en route à volonté des programmes de différenciation vers telle ou telle destinée. Ce bilan est extrêmement encourageant et vient vraiment à son heure. Je déplore seulement que le pouvoir immunogène (en cas d'allogreffes) et le cancer (risque de tératome), ces deux épées de Damoclès qu'il convient de considérer sous peine de retomber dans l'ornière du «hype without science », aient été insuffisamment évoqués par des orateurs sans doute soucieux de ne pas ternir leurs messages d'espoir. $\diamond$ Ex vivo veritas

Pour aider le lecteur à pleinement profiter des actes du Symposium FISH-ESC, je recommande une excellente revue générale, parue peu après la réunion.

Klimanskaya I, Rosenthal N, Lanza R. Derive and conquer : sourcing and differentiating stem cells for therapeutic applications. Nat Rev Drug Discov 2008 ; 7 : 131-42. 